\title{
Metamizole Versus Ibuprofen at Home After Day Surgery: a Double-blind Randomized Controlled Non-inferiority Trial
}

\section{K. Nijs, J. Dubois, S. Evers, L. Jamaer, J-P. Ory, B. Stessel}

Department of Anaesthesia and Intensive Care Unit, Jessa Hospital, Hasselt, Belgium

\section{Background and goal of the study}

Non-Steroidal Anti-Inflammatory Drugs (NSAID's) and paracetamol are the cornerstone of pain treatment after painful day surgery. Nevertheless, NSAID's are not always sufficiently effective, have numerous contraindications, and consequently are not suitable in up to $25 \%$ of all patients. Metamizole is a nonopioid compound with a favourable gastro-intestinal, cardiovascular and cerebrovascular profile compared to NSAID's. Hence, our aim is to assess if a combination of metamizole and paracetamol (MP) is non-inferior to a combination of ibuprofen and paracetamol (IP) to treat pain at home after painful day surgery.

\section{Methods}

A total of 200 patients undergoing elective hemorrhoid surgery, arthroscopic shoulder or knee surgery, or unilateral inguinal hernia repair in day setting were randomized to receive either MP $(n=100)$ or IP $(n=100)$. Patients in the MP-group (experimental arm) were instructed to take metamizole 1gr orally three times a day for four days and patients in the IP-group (control arm) were instructed to take ibuprofen $600 \mathrm{mg}$ orally three times a day for four days. All patients were treated with paracetamol $1 \mathrm{gr}$ orally four times a day during the entire study period.
Average postoperative pain intensity was assessed at postoperative day 0, 1, 2 and 3 using a Numeric Rating Scale (NRS) by telephone call. A difference in mean average pain NRS-score between MP and IP of 1 point or less is considered non-inferior. The outcome measure is analyzed on a intention-totreat (ITT) basis.

\section{Results and Discussion}

Despite the enrolment of 200 patients, only 196 patients were included in the ITT analysis because they did not receive the allocated intervention: three patients underwent a bilateral inguinal hernia repair and one patient was immediately excluded because of excessive preoperative opioid use. Average pain intensity scores and evaluation of non-inferiority of the analgesic efficacy of MP to IP are presented in table 1. MP is shown to be non-inferior at postoperative days (POD) 1 and 2. Before discharge and at POD 3, our results were inconclusive.

\section{Conclusion}

Our results suggest that the analgesic efficacy of MP is similar to IP for pain treatment at home after painful day surgery. We suggest MP to be a valuable alternative for IP in patients with a contraindication for NSAID.

\begin{tabular}{lcccc}
\hline \hline Average pain intensity & $\begin{array}{c}\text { Mean } \\
\text { Metamizole }+ \\
\text { Paracetamol }\end{array}$ & $\begin{array}{c}\text { Mean } \\
\text { Ibuprofen }+ \\
\text { Paracetamol }\end{array}$ & $\begin{array}{c}\text { Mean difference } \\
\text { and 95\% Cl }\end{array}$ & Inference \\
\hline ITT analysis & 3.03 & 2.49 & $0.541[-0.189 ; 1.270]$ & Inconclusive \\
Before discharge & 3.60 & 3.57 & $0.032[-0.647 ; 0.710]$ & Non-inferior \\
POD 1 & 3.22 & 3.27 & $-0.050[-0.758 ; 0.658]$ & Non-inferior \\
POD 2 & 3.18 & 2.95 & $0.236[-0.540 ; 1.011]$ & Inconclusive \\
POD 3 & & & & \\
\hline \hline
\end{tabular}

Table 1: Average pain intensity and evaluation of non-inferiority of the analgesic efficacy of metamizole and paracetamol to ibuprofen and paracetamol. The difference in mean numerical rating scale (NRS) scores for the imputed average pain intensity values between the two treatment groups (paracetamol and metamizole minus paracetamol and ibuprofen) and the resulting 95\% confidence intervals (Cl) are shown. A difference in mean NRS of less than 1 point is considered non-inferior, but the $95 \% \mathrm{Cl}$ that include the non-inferiority margin do not allow for a conclusive inference. POD: postoperative day; ITT: intention-to-treat. 